\title{
A Physical-Layer UHF RFID Tag Collision Resolution Based on Miller Code
}

\author{
Yu Zeng ${ }^{1,2}$ and Hongwei Ding $\mathbb{B}^{1}$ \\ ${ }^{1}$ School of Information Science \& Engineering, Yunnan University, 650091, China \\ ${ }^{2}$ School of Electrical \& Information Technology, Yunnan Minzu University, 650504, China \\ Correspondence should be addressed to Hongwei Ding; dhw1964@163.com
}

Received 27 November 2020; Revised 26 March 2021; Accepted 12 April 2021; Published 28 April 2021

Academic Editor: Luis Castedo

Copyright (C) 2021 Yu Zeng and Hongwei Ding. This is an open access article distributed under the Creative Commons Attribution License, which permits unrestricted use, distribution, and reproduction in any medium, provided the original work is properly cited.

\begin{abstract}
In an ultrahigh frequency (UHF) radio frequency identification (RFID) system, the throughput can be greatly improved by collision resolution on a physical layer when tags collide, and high-performance coding technology can improve the bit error rate (BER) performance of the physical-layer separation. Most of the traditional physical collision resolutions focus on the code with a single subcarrier. This paper pays more attention to Miller code with multiple subcarriers and proposes a novel physical-layer separation method based on the Miller code. In this method, the separated collision signals are multiplied by clock signals with the same frequency as the subcarrier to complete the frequency shift. And then, a coherent demodulation and a low-pass filter are used to remove high-frequency separation noise. In the simulation, the Miller code with more subcarriers has lower BER than FM0 code with a single carrier. Especially when Miller 8 is selected, the separation efficiency and BER performance of the proposed method are $4 \mathrm{~dB}$ higher than those of the traditional XOR method at lower SNR.
\end{abstract}

\section{Introduction}

Wireless radio frequency identification (RFID) is an automatic identification technology that uses wireless radio frequencies to conduct noncontact data communication through electromagnetic fields. Because ultrahigh-frequency (UHF) RFID has the advantages of fast and efficient identification of the target objects $[1,2]$, it has become one of the key sensor technologies of the Internet of Things. UHF RFID usually communicates on a wireless shared channel. Thus, when readers read multiple tags simultaneously, signal collision between tags will be inevitable.

Tag collision is usually resolved at a Media Access Control (MAC) layer. The currently popular UHF RFID standard-EPC C1 Gen2 [3] uses a MAC layer protocol to resolve tag collisions. The basic idea is that a tag can randomly select the time slot via its counter. However, if two or more than two tags select the same time slot, the time slot is still an invalid collision time slot. Therefore, the identification efficiency is not optimal. In recent years, a physical layer separation technology can identify tags in collision time slots and can greatly improve the identification efficiency [4]. The technology requires the separation of collided signals before the decoding. However, the separation can cause separation errors which will affect the decoding performance. So, it is essential to improve collision resolution performance on the physical layer by encoding methods to remove the separation errors [5].

The encoding method in EPC C1 Gen2 adopts FM0 and Miller codes [3, 6]. For the FM0 code, whether a symbol is 0 or 1 is determined by whether there is a phase jump during a symbol period. Since FM0 cannot require a rigor time synchronization like in a matched filter, it can resist the frequency drift commonly in wireless communication systems. However, if the collision signals are separated on the physical layer, the separated signals will have some separation errors. The method of phase jump will increase the bit error rate (BER). Unfortunately, many existing physical layer collision separation algorithms use the FM0 code [7-11].

Compared with the FM0 code, the Miller code has some subcarriers and usually has a higher frequency than that of the FM0 code. High-frequency noise can be removed with 

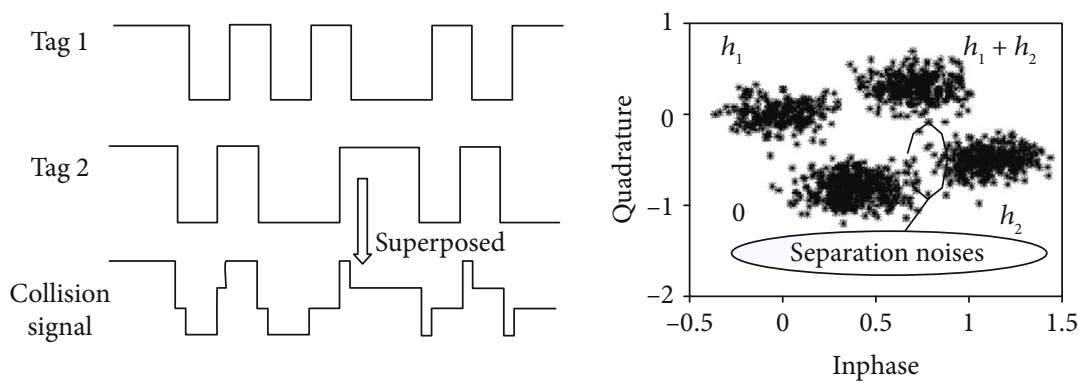

FIGURE 1: Separation of two collision signals.

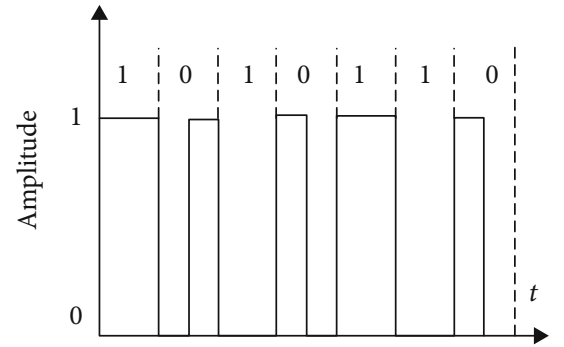

(a) FM0 coding without separation noise

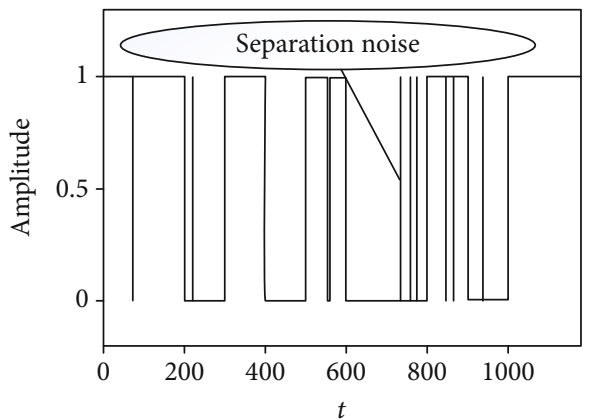

(b) FM0 coding with separation noise

Figure 2: FM0 coding with and without separation noise.

the corresponding removing of high-frequency subcarriers. Thus, the Miller code performs better on high-frequency noises. Traditional Miller decoding uses XOR operation to remove subcarriers [12-15], which can resist the frequency drift that exists in the FM0 code. However, in the physical layer separation, the Miller code still cannot eliminate the separation noise after the XOR operation.

In our early work in [12], we only adopt traditional decoding methods to evaluate the performance of Miller and FM0 codes and there are no decoding methods proposed. This paper proposes a physical-layer separation miller decoding (PMD) method to resolve the tag collision. It has the following contributions. First, it proposes a new method for decoding the Miller code by spectrum shifting. In this method, the separated baseband signal is multiplied by two subcarriers whose phases are different by $\mathrm{pi} / 2$, and then decoded after low-pass filter and envelope detection. Compared with the traditional XOR decoding method, the proposed method can eliminate more separation errors. Second, the proposed method has better performance of BER under low signal-to-noise ratio (SNR). It can be applied to improve the performance of the tag anticollision when the reader's transmit power is lower or noise interference is larger. The experimental results show that when the decoding method proposed in this paper is used, the Miller code with higher-frequency subcarriers has better separation efficiency, e.g., Miller 8. The BER performance of this method is $4 \mathrm{~dB}$ higher than that of the traditional decoding method when the same symbol rate is adopted.

\section{Related Work}

In an UHF RFID system, when multiple tags collide at a reader, the collided signal received by the reader is a superposed signal of multiple tags. Figure 1(a) shows the superposed signal after two tag collisions, where these tags use a unipolar code, and the signal fading and symbol periods of two tags are different. Generally, the collided signal of multiple tags can be separated by clustering [16-19]. Figure 1(b) shows the mapped constellation of the collided signal in the phase/quadrature (IQ) plane [17]. If the fading coefficients of these two tags are $h_{1}$ and $h_{2}$, then the centers of the four clusters in the figure are $0, h_{1}, h_{2}$, and $h_{1}+h_{2}$. Thus, the signal points that belong to different clusters can be separated into $(0,0),(0,1),(1,0)$, and $(1,1)$, respectively.

The separated tag signals are only encoded signals, and the tag IDs can be obtained only after the decoding. In a UHF RFID system, EPC C1 Gen2 recommends FM0 and Miller codes $[3,6]$. In FM0, there is a phase jump in the middle of symbol 0 , there is no jump in the middle of symbol 1 , and there must also be a jump between symbols. Therefore, in FM0 decoding, we only judge whether there is a jump near the midpoint of the symbol period. Figure 2(a) shows an example of FM0 coding with ID 1010110. However, after separation is performed on a physical layer, the decoding method may generate errors due to the separation noise. The separation noise generated by the clustering in Figure 1 may produce the separated FM0 signals shown in Figure 2(b). As can be seen from Figure 2(b), separation noise is generated around $t=600$. From the FM0 encoding rule, 


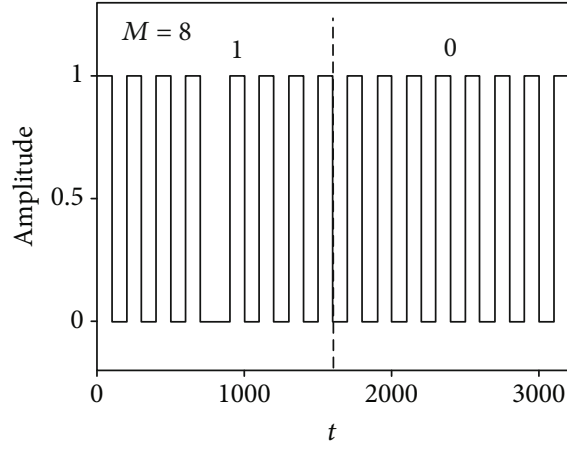

(a) Miller coding without separation noise

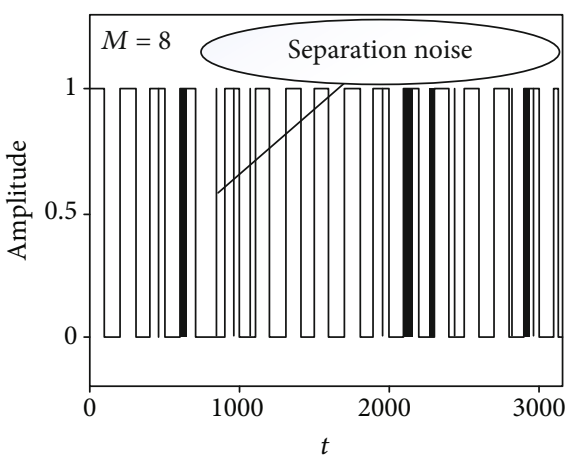

(b) Miller coding with separation noise

FIGURE 3: Miller coding with and without separation noise.

the signal will be misjudged as 0 since this is the midpoint of a symbol period and a phase jump has occurred. A simple solution for the error is to use a matched filter to eliminate the separation noise [20]. However, the matched filter eliminates noise by adopting a maximum SNR criterion. The BER will increase with the separation noise.

Another code recommended by EPC C1 Gen2 is the Miller code, whose steps are as follows. A phase jump occurs at the midpoint of symbol 1, and no jumps occur between the symbol 1 and its previous symbol; no phase jumps occur at the midpoint of a symbol 0 and between the symbol 0 and its previous symbol 1, and a jump occurs between the symbol 0 and its previous symbol 0. Figure 3(a) shows a Miller 8 signal without separation noise. If the Miller 8 signal is a separated one, the result may be that shown in Figure 3(b). Due to the separation noise, symbol errors are likely to occur if we only judge whether there is a jump near the midpoint of the symbol, like in FM0 decoding. However, compared with the FM0 code, the Miller code has more subcarriers. From Figure 3(b), we can find that the separation noise is actually superimposed on the subcarriers with high frequency. In the spectrum analysis, when a low-pass filter is used to filter out subcarriers, the highfrequency separation noise can actually be removed. Thus, the influence of separation noise on decoding can be reduced. Unfortunately, the FM0 code is applied to many existing physical-layer collision separation algorithms. This paper will apply the Miller code to improve the performance of physicallayer separation. In addition, there is a parallel decoding technique in RFID decoding methods [21,22], which has also been widely used in collision resolution. Since this paper mainly focuses on the Miller and FM0 code in EPC C1 Gen2, we will not introduce the technique too much.

\section{Problem Description}

Traditionally, Miller decoding generates a clock signal with the same frequency as the subcarrier and then executes the XOR operation on the clock signal and a received Miller code [1214]. Figure 4(a) shows an example of a Miller 2 code with the subcarrier removing. From the figure, the XOR operation can remove the high-frequency subcarrier. After the subcarrier is removed, the signal can be decoded by whether there is a phase jump during symbol 1 and no jump during symbol 0 .
The above decoding method is usually applied to the case where UHF RFID tags do not collide. When the physicallayer separation algorithm is used, the separated Miller signal will have separation noise, as shown in Figure 4(b). Even after the XOR operation with a clock signal, the separation noise still exists. Therefore, the decoding method does not reduce the separation noise even after the subcarrier is removed.

Figure 4(c) shows the algorithm flowchart of this paper. When multiple tags send signals to the reader, the received signal is a superposition of collided tags. The reader first adopts the ALOHA method to resolve the tag collision. It uses the physical layer decoding to recover the collided tags in a time slot. The collided tags are sampled and separated, and the proposed PMD decoding is performed, respectively. The next section will introduce PMD decoding steps in detail.

\section{Methods}

4.1. Overview. This section first explains how our multiplying operation eliminates the separation noise. When the clock signal at 0 is multiplied by the Miller code with the separation noise, thus, the separation noise will be 0 , as shown in the circle in Figure 5(a). On the other hand, any signal multiplied by 1 will not change. In this case, the separation noise during the clock signal at 1 cannot be changed, as shown in the diamond in Figure 5(a). Consider another type of multiplication. Invert the phase of the clock signal, and then, multiply it with the Miller code with the separation noise. The separation noise during the original clock signal at 1 will also be changed into 0, as shown in the circle in Figure 5(b). Of course, the separation noise during the inverted clock signal at 1 cannot be removed, as shown in the diamond in Figure 5(b). Therefore, these two multiplied signals need to be synthesized to obtain a final signal.

In addition, from Figure 5, the subcarrier is not completely removed after multiplication with the clock signal. However, in the spectrum analysis, multiplying the Miller code with the same frequency clock signal as the subcarriers is actually a frequency shift. That is, the frequency shifts to the low frequency that the original symbol has and to a frequency higher than the original symbol. Therefore, if a reasonable cutoff frequency is set for a low-pass filter and let the multiplied signals pass through the filter, we can 


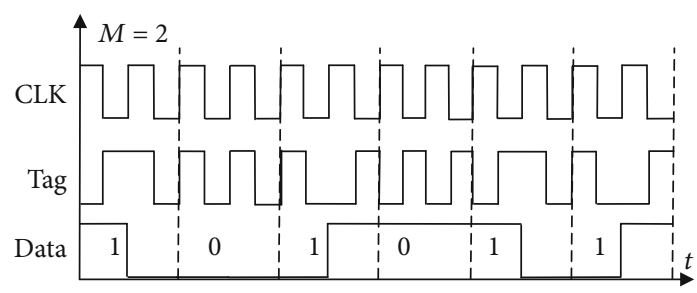

(a) Remove subcarrier without separation noise by XOR

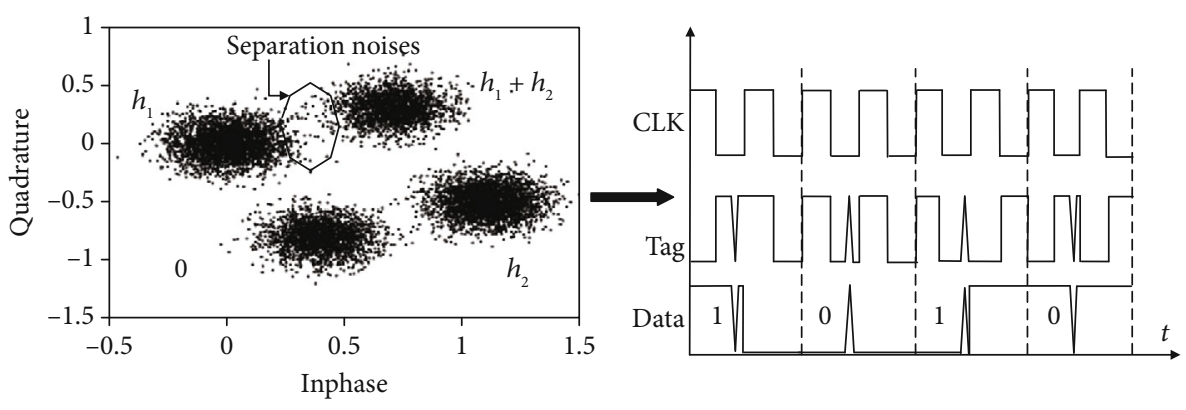

(b) Remove subcarrier with separation noise by XOR

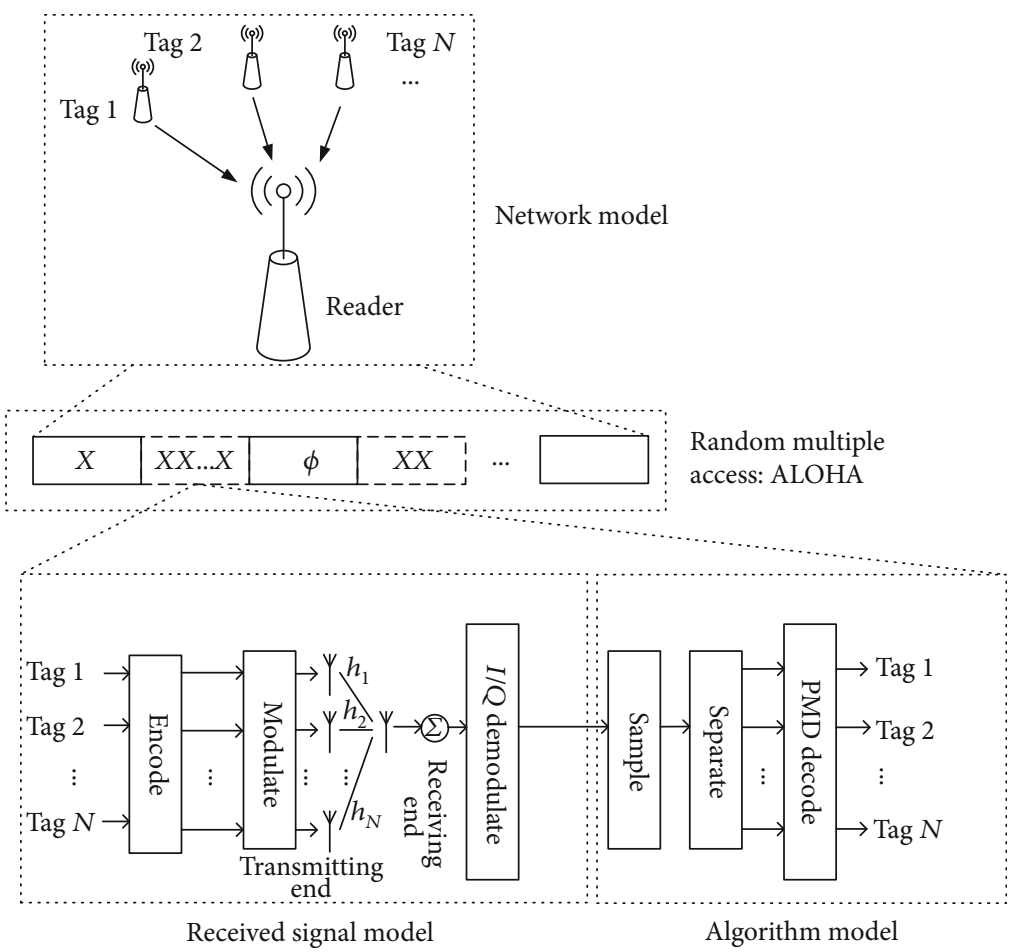

(c) Flowchart of the proposed algorithm

FIGURE 4: Miller decoding and flowchart.

obtain the original symbol. Next, we will discuss it in more detail.

4.2. Multiplication and Low-Pass Filter. To find the cutoff frequency of the low-pass filter after the Miller code is multiplied by the clock signal, we firstly need to write the mathematical expression of the Miller code, as shown in Figure 6.

From the Miller coding rule, symbols $y_{i} \in\{0,1\}, i=0,1$ $, 2, \cdots$, with a period $2 T$ have four basis functions $S_{0}, S_{1}, S_{2}$, and $S_{3}$ as shown in Figure 6(b). Then, the $i$ th symbol can be expressed as two basis functions

$$
d_{k}, d_{k+1} \in\{1,-1\}, \quad k=2 i .
$$

And its waveform function can be expressed as

$$
s(t)=\sum_{k} d_{k} g(t-k T)
$$




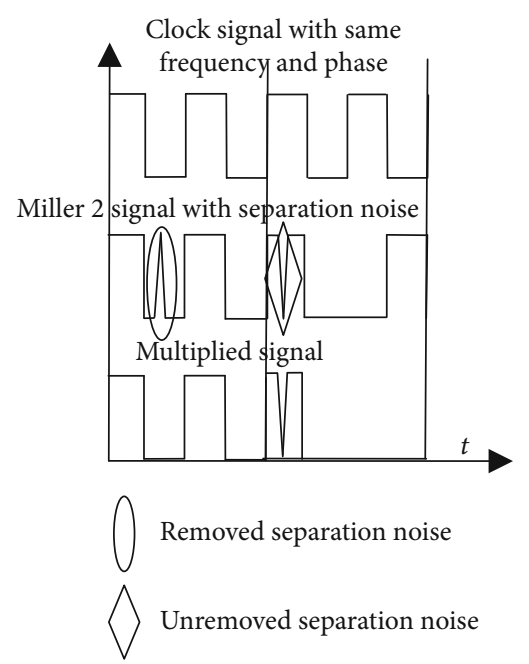

(a) Multiplied by same phase clock signal to remove separation noise

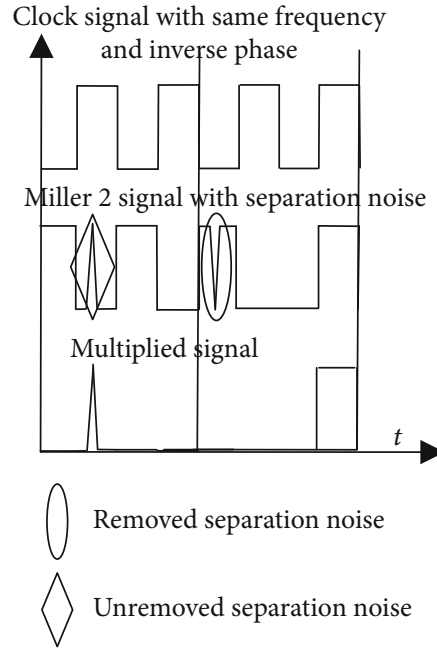

(b) Multiplied by inverse phase clock signal to remove separation noise

Figure 5: An example for multiplication to eliminate separation noise in Miller 2 code.

(a) Binary symbol sequence $y_{i}, i=0,1, \ldots$

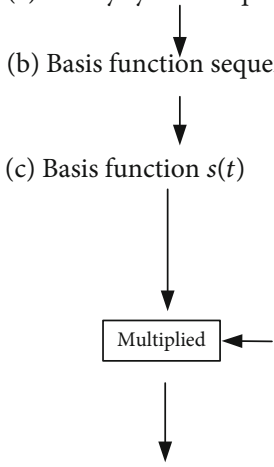

(d) Basis function multiplied by subcarrier (c) Subcarrier $f(m, t)$
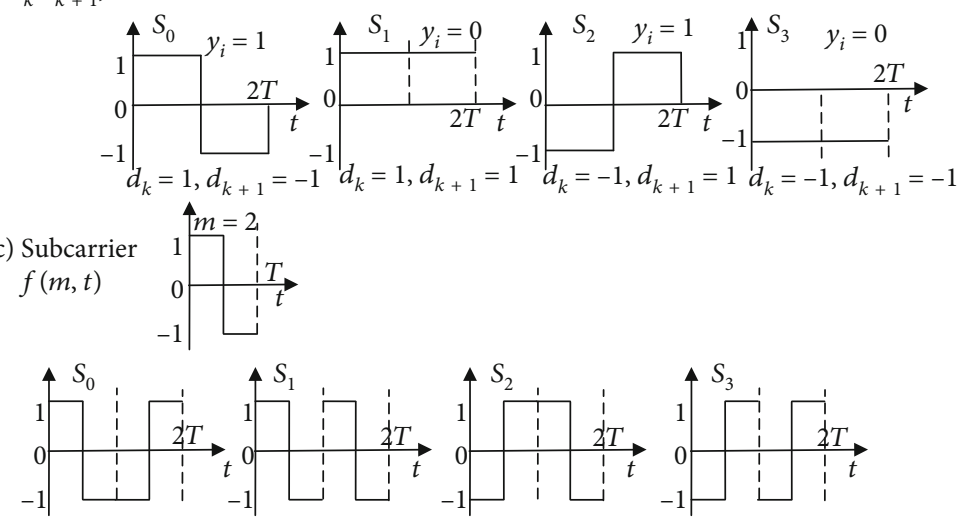

(e) Normalized to $[0,1]$
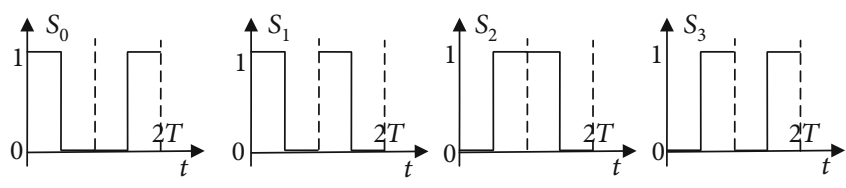

(f) Combined

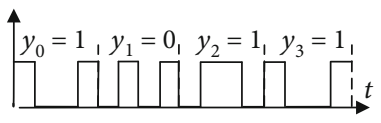

Figure 6: Mathematical expression of Miller 2 code.

where

$$
g(t)= \begin{cases}1, & t \in[0, T] \\ 0, & \text { elsewise }\end{cases}
$$

In Figure 6(c), the subcarrier with only one period can be Fourier-series expanded into

$$
f(m, t)=\left\{\begin{array}{l}
\frac{4}{\pi} \sum_{n=0}^{+\infty} \frac{1}{2 n+1} \sin \left[(2 n+1) \frac{m \omega_{c} t}{2}\right], \quad t \in[0, T], \\
0, \quad \text { elsewise, }
\end{array}\right.
$$

where $\omega_{c}=2 \pi / T$ and $m=2,4,8$ denote Miller 2, Miller 4, and Miller 8, respectively. Multiplying (4) by (2), the subcarrier of the base function code in Figure 6(d) can be expressed 


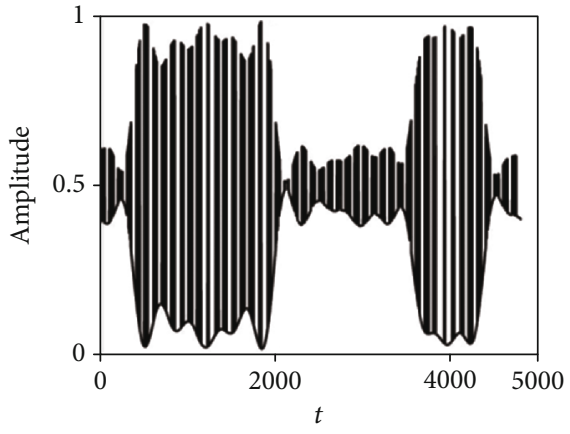

(a) Low-pass filtered signal

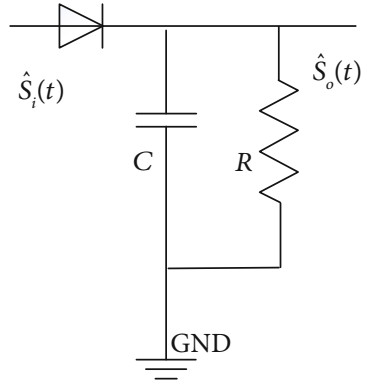

(c) Envelope shaping circuit

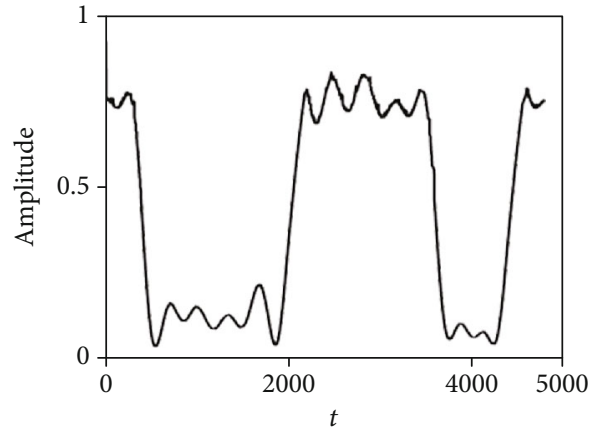

(b) Bottom envelope

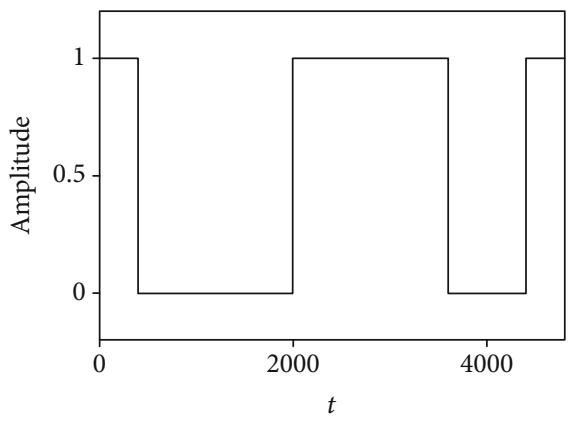

(d) Basis function waveform

Figure 7: Envelope shaping.

by

$$
x(t)=\sum_{k} s(t) f(m, t-k T)
$$

Since the Miller code is a binary unipolar code as shown in Figure 6(e), after normalizing $(5)$ to $[0,1]$, the final waveform of Figure 6(f) is

$$
x(t)=\frac{1}{2} \sum_{k} s(t) f(m, t-k T)+\frac{1}{2} .
$$

Next, we will find the cutoff frequency of the low-pass filter through the expression of the Miller code. Equation (6) multiplied by the clock signal with the same frequency as the subcarriers will have

$$
Z(t)=x(t) \sum_{k} f(m, t-k T)
$$

From (4), (7), and a trigonometric product-to-sum identity, the set of angular frequencies for (7) can be expressed as

$$
\omega_{z}=\left\{0, \Omega_{c}, 2 \Omega_{c}, 3 \Omega_{c}, \cdots\right\}
$$

where $\Omega_{c}=m \omega_{c} / 2$. Therefore, if the cutoff frequency is set between $\left[0, \Omega_{c}\right]$, then the basis function of the Miller code can be obtained through the low-pass filter with the cutoff frequency, i.e.,

$$
\widehat{s}(t)=\operatorname{LF}[Z(t)]
$$

where $\operatorname{LF}(\cdot)$ represents the low-pass filter. Then, the basis function code $d_{k}$ is obtained from $\widehat{s}(t)$, and thus, the original symbol $y_{i}$ is obtained from $d_{k}$ through the Miller coding rule.

Note that the Miller code can also be multiplied by an inverse-phase clock signal, i.e.,

$$
Z(t)=x(t)[-f(m, t-k T)]
$$

Then, the signal in (10) through the filter in (9) will have another basis function code $d_{k}$.

4.3. Shaping, Synthesis, and Decoding. Theoretically, the cutoff frequency of the low-pass filter set between $\left[0, \Omega_{c}\right]$ can eliminate the high-frequency subcarrier and high-frequency separation noise. In applications, however, some highfrequency components close to the cutoff frequency cannot be completely removed, as shown in Figure 7(a). If we set the cutoff frequency lower, it will affect the filtered basis function code. Here, we will use envelope shaping to further eliminate high-frequency noise.

It can be seen from Figure 7(a) that although the filtered signal has high-frequency components, its bottom envelope is consistent with the waveform of the basis function code in (d). Consider the circuit in (c) to realize the envelope shaping [20, 23]. Then, the bottom envelope can be expressed as 


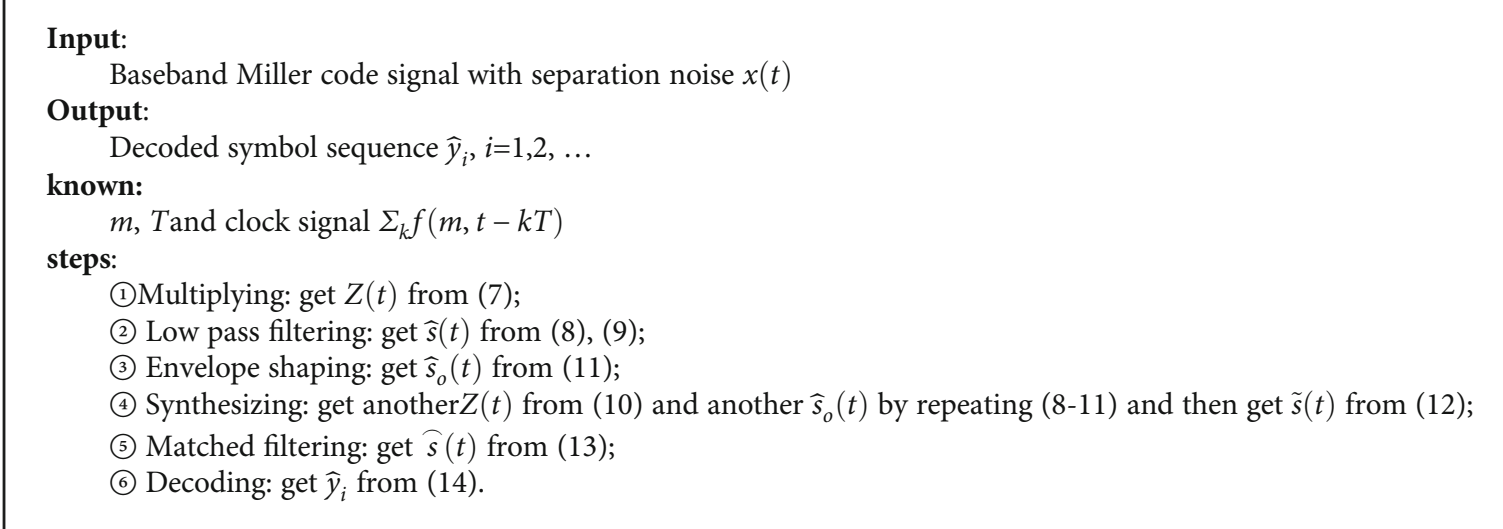

Algorithm 1: Steps of PMD algorithm.

$$
\widehat{s}_{o}(t)= \begin{cases}-\widehat{s}_{i}(t), & \text { if } \widehat{s}_{i}(t)>\widehat{s}_{o}(t-\Delta t), \\ -R C \frac{\Delta \widehat{s}_{o}(t)}{\Delta t}, & \text { if } \widehat{s}_{i}(t)<\widehat{s}_{o}(t-\Delta t),\end{cases}
$$

where $R$ denotes a resistance, $C$ denotes a capacitance, $\widehat{s}_{i}(t)=$ $\widehat{s}(t)$, and $\Delta$ is a very small value.

Multiplying the same phase clock signal can only remove half of the separation noise. Similarly, multiplying the inverse phase clock signal can only remove another half of the noise. For this, the two envelope shaping signals are synthesized and averaged to obtain

$$
\tilde{s}(t)=\frac{1}{2}\left[\widehat{s}_{o 1}(t)+\widehat{s}_{o 2}(t)\right]
$$

where $\widehat{s}_{o 1}(t)$ and $\widehat{s}_{o 2}(t)$ are the signals after multiplying the same and inverse phase clock signals via envelope shaping in (11).

To further eliminate noise, the synthesized signal is passed through a matched filter to obtain [24-26]

$$
\widehat{\mathcal{s}}(t)=\tilde{s}(t) \otimes h(t)
$$

where $\otimes$ is a convolution operation and $h(t)$ is a matched filter. After sampling $\widehat{s}(t)$ at $k T$, we decide to get its basis function symbol $\widehat{d}_{k}$. From the Miller code rule, the transmitted symbol is finally

$$
\widehat{y}_{i}=D\left(\widehat{d}_{k}, \widehat{d}_{k+1}\right), \quad i=2 k, k=0,1, \cdots
$$

where $D(\cdot)$ represents the Miller decoding rule, i.e., no phase jumps during symbol 0 and a jump during symbol 1 .

Algorithm steps

Finally, we list the steps of the above decoding in Algorithm 1.

\section{Results and Discussion}

5.1. Experiment Setup. In the experiment, there is a reader and two collision tags, and the experimental results are all independently repeated 500 times and then averaged. The values of some system parameters are from EPC C1 Gen2 standard [3], and the other ones refer to the literature [712]. The parameters are as follows:

(i) Channel: in the UHF system, due to the small length of the tag data, the communication time is also short. The channel can be regarded as a linear timeinvariant channel. Because of the variation of tags' location, in addition, the channel fading coefficient will be different

(ii) Coding: EPC C1 Gen 2 standard provides two codes, FM0 and Miller. This experiment adopts FM0, Miller 4, and Miller 8

(iii) Symbol block length: EPC C1 Gen 2 standard specifies that RN16 is sent firstly with a code length of 16 after a tag response, so the block length is set as 16

(iv) Reader antenna: one antenna is used to perform the data transmission and reception of the reader

(v) Symbol rate: EPC C1 Gen 2 standard defines that a link frequency range is from $40 \mathrm{kHz}$ to $320 \mathrm{kHz}$. This experiment adopts $50 \mathrm{kHz}$

(vi) Sampling frequency: low sampling rate causes too few signal points in one symbol period, which is not conducive to signal separation. The actual signal sampling can be realized by software radio or oscilloscope, and its sampling frequency can reach about $600 \mathrm{MHz}$. In this experiment, 25.6 MHz can be used to ensure the algorithm performance

In the experiment, the simulation of collision tag separation is performed from literature [7, 8]. First, the channel coefficients are estimated by a least square method through the preamble, and the four cluster centers of the collision signal on an IQ map are determined. Then, map the collision signals to the IQ plane, calculate the Euclidean distance from each signal sample to the four cluster centers, and determine the cluster to which the sample belongs based on the minimum distance. Finally, the tag signal separation is completed by the belonging cluster. 
TABle 1: Parameters of Mille4PMD and Miller8PMD.

\begin{tabular}{ll}
\hline Parameter & \multicolumn{1}{c}{ Description } \\
\hline Envelope detection & $\begin{array}{r}R C=10 \mathrm{~s} \text { (the value is determined by the signal frequency. In applications, } \\
\text { we can choose an optimal value after trying multiple values.) }\end{array}$ \\
\hline $\begin{array}{l}\text { Low-pass filter cutoff } \\
\text { frequency }\end{array}$ & $\begin{array}{r}\text { Mille8PMD uses a fixed cutoff frequency of } 16 \mathrm{kHz} \text {. (The choice of } 16 \mathrm{kHz} \text { is from the simulation results of } \\
\text { frequency; when the signal-to-noise ratio is }-10,-8,-6,-4,-2,0,2,4,6 \text {, and } 8 \mathrm{~dB} \text {, the cutoff frequencies are } 15,15, \\
14,20,19,16,21,26,46, \text { and } 19 \mathrm{kHz}, \text { respectively, seen in subsection Dynamic Cutoff Frequency for details }\end{array}$ \\
\hline
\end{tabular}

This experiment will give the performance comparison of the following decoding methods:

(i) Miller4XOR and Miller8XOR: Miller 4 and Miller 8 are applied to encoding, and XOR operation $[13,19]$ is applied to decoding

(ii) Mille4PMD and Miller8PMD: Miller 4 and Miller 8 are applied to encoding, and Algorithm 1 is applied to decoding. The parameters of the decoding algorithms are in Table 1

(iii) FM0: FM0 is used to encode, the separated collision signals are passed through matched filtering, and the filtered signals are decoded by whether there is a phase jump during a symbol [12].

In the simulation experiment, we will give the decoding efficiency and BER change of each algorithm with SNR, which is defined as

$$
\mathrm{SNR}=10 \lg \frac{P_{\mathrm{s}}}{P_{\mathrm{n}}},
$$

where $P_{\mathrm{s}}$ is the average power of the collision signals after IQ demodulation and $P_{\mathrm{n}}$ is the noise power. The decoding efficiency of the $i$ th tag is defined as follows:

$$
\eta_{i}=\frac{n_{i}}{n_{\mathrm{t}}} \times 100 \%
$$

where $n_{\mathrm{t}}$ is the total number of collision tags and $n_{i}$ is the number of the $i$ th tag successfully decoded from $n_{\mathrm{t}}$ collision tags. In the experiment, only if there is no one-bit decoding error in an RN16 sent by a tag can the tag be regarded as a successful decoding tag. Higher decoding efficiency means better decoding performance. The decoding BER of the $i$ th tag is defined as

$$
R_{i}=\frac{c_{i}}{C_{i}}
$$

where $c_{i}$ is the number of bit errors that occur when decoding the $i$ th tag, and $C_{i}$ is the total number of bits of the $i$ th tag. Smaller BER means better decoding performance.

5.2. Decoding Efficiency. Figures 10 and 11 show the decoding efficiency curves of the first tag and the second tag, respectively. In general, the change trend of the separation effi-

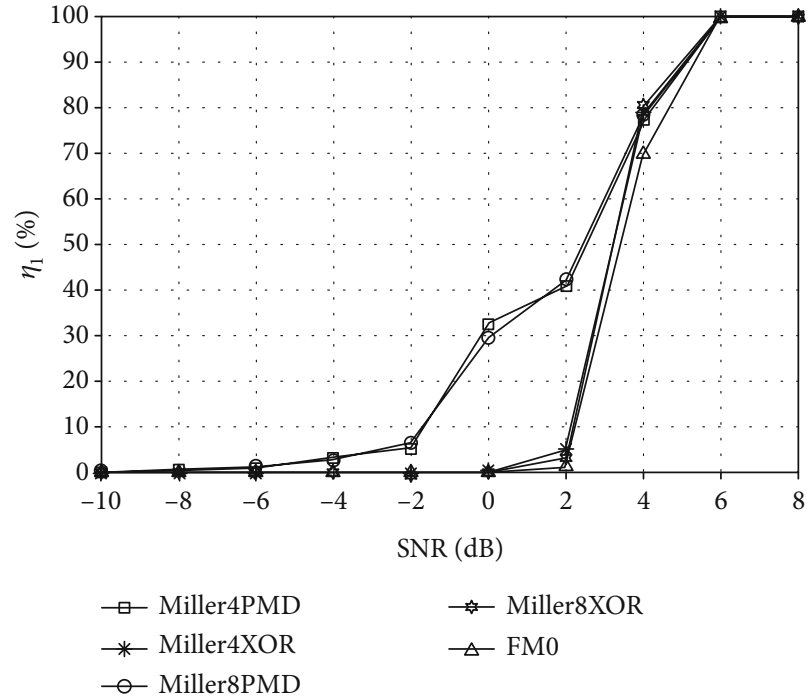

FIGURE 8: PMD algorithm's cutoff frequency at the lowest BER.

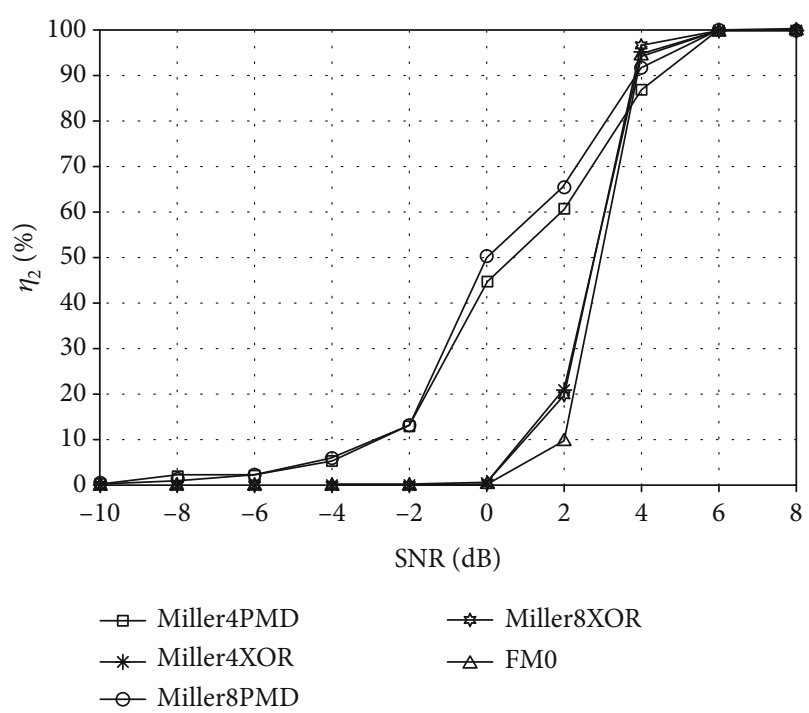

FIGURE 9: BER of Miller8PMD with different cutoff frequencies.

ciency curves of the two tags is the same, so there is no significant difference in the decoding results of each decoding algorithm for each tag. First, when SNR is between $-10 \mathrm{~dB}$ and $4 \mathrm{~dB}$, the decoding efficiency of PMD decoding for both Miller8 and Miller4 is higher than the traditional XOR algorithm. Therefore, when the number of subcarriers of the 


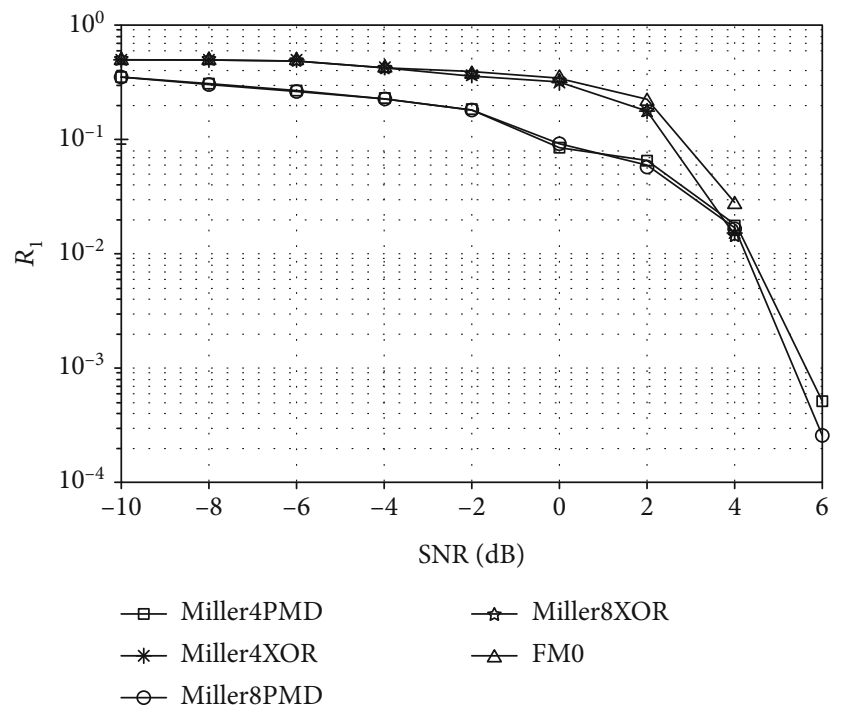

Figure 10: Decoding efficiency of the first tag.

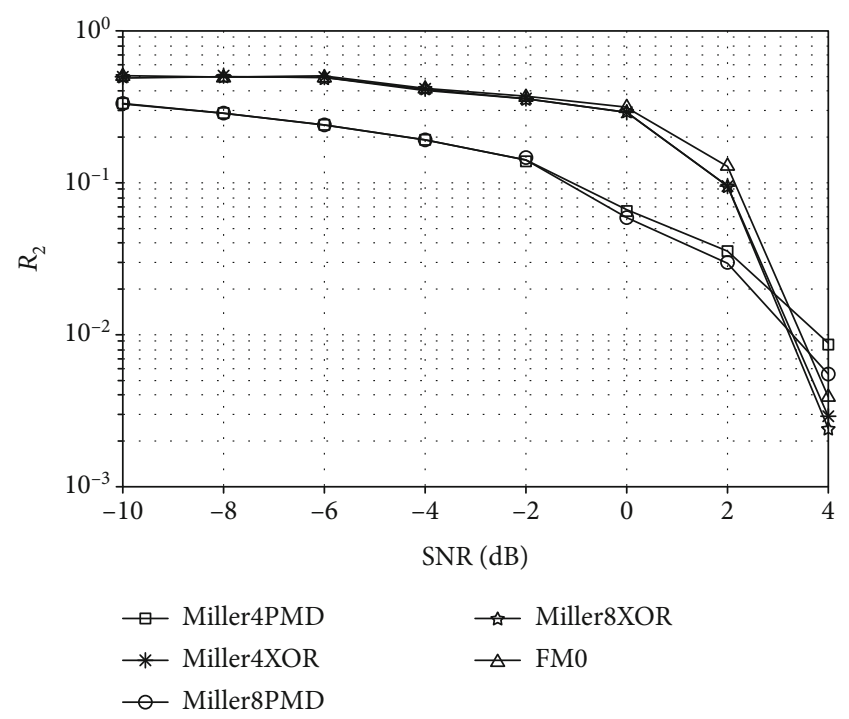

Figure 11: Decoding efficiency of the second tag.

Miller code is the same, the decoding efficiency of PMD is better. Second, the PMD decoding efficiency can be improved by more subcarriers. When SNR is between $-2 \mathrm{~dB}$ and $2 \mathrm{~dB}$, the PMD decoding efficiency curve performs better than the FM0 curve with single subcarrier by about $4 \mathrm{~dB}$. On the other hand, the traditional XOR Miller decoding curve coincides with the single-type FM0 curve, and its XOR decoding method does not take advantage of more subcarriers to improve its efficiency. Similarly, the Miller8PMD performs better than Miller4PMD as it has more subcarriers. Finally, the decoding advantage of the algorithm in this paper is mainly reflected under lower SNR because the decoding efficiency of each algorithm can reach $100 \%$ when SNR exceeds $6 \mathrm{~dB}$.

5.3. Decoding BER. Figures 12 and 13 show the BER curves of the traditional and the proposed methods when two tag sig-

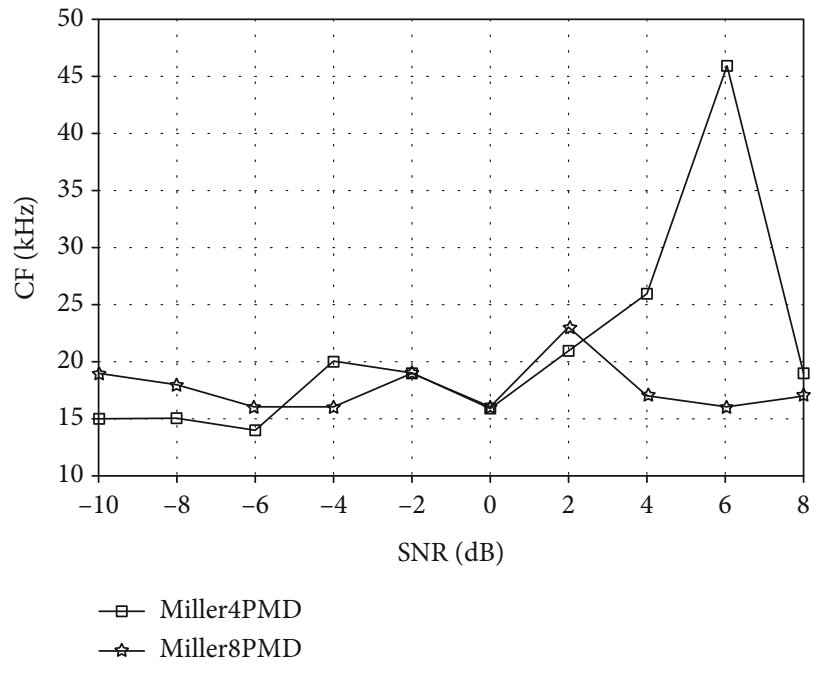

FIGURE 12: Decoding BER of the first tag.

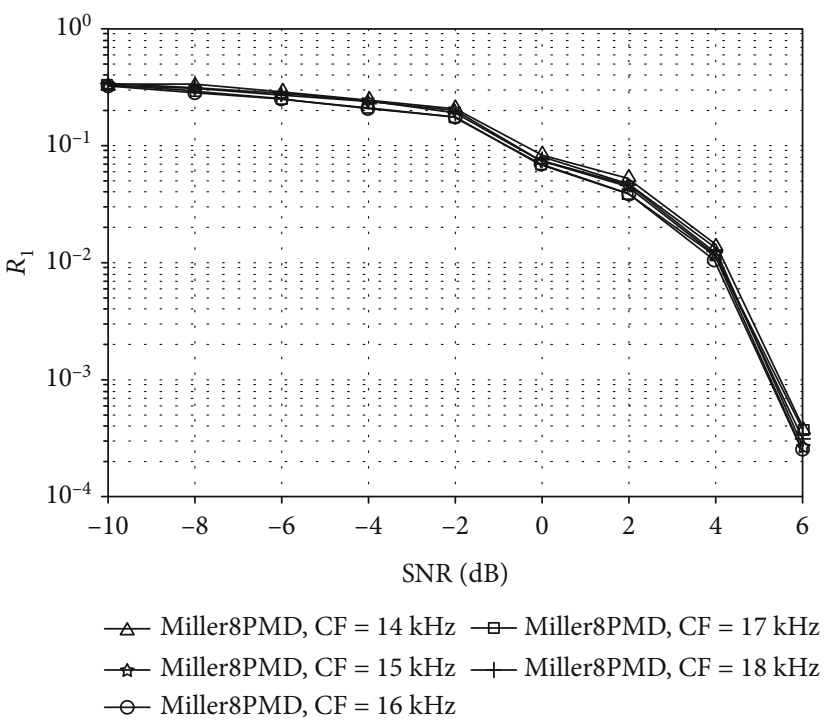

FIgUre 13: Decoding BER of the second tag

nals collide under different SNRs. First, since there will be more separation errors when SNR is low, the PMD algorithm shows better performance owing to more separation errors eliminated. When SNR is between $-6 \mathrm{~dB}$ and $2 \mathrm{~dB}$, PMD's BER is better than the traditional XOR decoding methods by about $4 \mathrm{~dB}$. Second, when SNR is higher than $4 \mathrm{~dB}$, PMD's BER is slightly lower than the traditional XOR decoding methods. However, the system efficiency is mainly determined by the decoding efficiency. Note that in the decoding rate curves of Figures 10 and 11, the decoding efficiency of the PMD algorithm is close to $100 \%$ when SNR is greater than $4 \mathrm{~dB}$. PMD's efficiency is the same as the traditional XOR algorithm. Thus, the difference between their BERs will not cause the difference between their final system performance. In addition, since Miller8PMD's BER is slightly higher than that of Miller4PMD, it is a good way for PMD to increase the subcarriers to improve the BER performance. 
5.4. Dynamic Cutoff Frequency. This subsection will give the influence of the low-pass cutoff frequency on the proposed algorithm. Figure 8 shows the cutoff frequency at the lowest BER of the proposed algorithm under different SNRs, i.e., the optimal cutoff frequency curves (note that the optimal frequency results for two tags are the same, so only one curve for the tags are given). As can be seen from the figure, Miller4PMD's optimal cutoff frequency range is about 15$45 \mathrm{kHz}$. The values fluctuate larger. Therefore, to obtain better decoding performance, a dynamic cutoff frequency is selected. For Miller8PMD, the optimal cutoff frequency range is $15-23 \mathrm{kHz}$, which fluctuates smaller. Thus, an average fixed cutoff frequency can be considered. This result also shows that when the number of subcarriers is smaller, the decoding is greatly affected by the separation noise, so the optimal cutoff frequency is likely to fluctuate.

Figure 9 shows the BER curves of Miller8PMD at the cutoff frequency of $14,15,16,17$, and $18 \mathrm{kHz}$, respectively. It can be seen from the figure that these four curves roughly coincide. The results show that if Miller8PMD selects a fixed optimal cutoff frequency, the above frequency values will produce similar results. From the simulation results of Figures 8 and 9, the optimal cutoff frequencies of Miller8PMD fluctuate slightly with SNR, about from 15 to $20 \mathrm{kHz}$. On the contrary, the fluctuation of Miller4PMD is relatively large. Therefore, it is recommended to choose Miller8 with more subcarriers and set the cutoff frequency to about $16 \mathrm{kHz}$.

In addition, since we apply a spectrum shifting method to eliminate the separation error, the hardware requires some circuits such as multiplication, low-pass filtering, and envelope shaping. On the other hand, as the traditional XOR decoding algorithm only needs an XOR circuit to complete the decoding, so the complexity of the proposed algorithm is higher than the XOR method. In addition, the proposed algorithm needs to resolve the tag collision on a physical layer, and the collision signals need to be separated first. Therefore, it also has higher complexity than other state-ofthe-art collision-resolution schemes on an MAC layer [14]. However, from the experimental results, the proposed algorithm trades more complexity for better performance.

\section{Conclusions}

This paper studies the Miller decoding performance of UHF RFID collision tags and proposes a Miller decoding method that can be applied to the collision signal separation on a physical layer. Compared with the traditional Miller decoding, the proposed decoding method can eliminate more separation errors at lower SNR. From the results of numerical experiments, when SNR is between $-2 \mathrm{~dB}$ and $2 \mathrm{~dB}$, the decoding efficiency and BER of Miller 8 and Miller 4 in the proposed method are better than those in the traditional method by about $4 \mathrm{~dB}$. Furthermore, when SNR exceeds $4 \mathrm{~dB}$, this method has similar decoding efficiency and BER performance to traditional algorithms. Besides, when SNR is greater than $2 \mathrm{~dB}$, the decoding efficiency and BER performance of Miller 8 decoding in this method are better than those of Miller 4, so appropriately increasing the number of subcarriers in the Miller code can improve the decoding performance. Finally, the setting of the low-pass cutoff frequency is also an important factor affecting the performance of the Miller decoding method in this paper. Miller 4 needs to set a dynamic cutoff frequency to obtain better decoding performance, while Miller 8 only needs to set a fixed cutoff frequency.

\section{Data Availability}

The research data used to support the findings of this study are included within this paper.

\section{Conflicts of Interest}

The authors declare that there is no conflict of interest regarding the publication of this paper.

\section{Acknowledgments}

The research and publication of this article were funded by the National Natural Science Foundation of China (61461053, 61461054, 61762093) and the Key Application and Basic Research Fund of Yunnan Province under Grant 2018FA036.

\section{References}

[1] K. Finkenzeller, "RFID Handbook," in Radio-Frequency Identification Fundamentals and Applications, Wiley, London, U.K, 2nd edition, 2003.

[2] J. Zhang, Y. Lyu, J. Patton, S. C. G. Periaswamy, T. Roppel, and BFVP, "A probabilistic UHF RFID tag localization algorithm using Bayesian filter and a variable power RFID model," IEEE Transactions on Industrial Electronics, vol. 65, no. 10, pp. 8250-8259, 2018.

[3] EPC Global, EPC Radio Frequency Identity Protocols Class-1 Generation-2 UHF RFID Protocol for Communications at 860 MHz-960 MHz, EPCglobal INC, 2013.

[4] N. T. Dang and V. V. Mai, "A PHY/MAC cross-layer analysis for IEEE 802.15.7 uplink visible local area network," IEEE Photonics Journal, vol. 11, no. 3, 2019.

[5] Y. Zhang, L. T. Yang, and J. Chen, RFID and Sensor Networks: Architectures, Protocols, Security and Integrations, CRC Press, United States, 2009.

[6] International Standard ISO/IEC 18000-6C, Information Technology Radio Frequency Identification (RFID) for Item Management Part 6: Parameters for Air Interface Communications at $860 \mathrm{MHz}$ to $960 \mathrm{MHz}, 2005$.

[7] H. Duan, H. Wu, Y. Zeng, and Y. Chen, "LS channel estimation and signal separation for UHF RFID tag collision recovery on the physical layer," Sensors, vol. 16, no. 4, pp. 442-451, 2016.

[8] J. Li, H. Wu, and Y. Zeng, "Recovery of collided RFID tags with frequency drift on physical layer," IEEE/CAA Journal of Automatica Sinica, vol. 7, no. 6, pp. 1593-1603, 2020.

[9] H. Wu, Y. Zeng, J. Feng, and Y. Gu, "Binary tree slotted aloha for passive RFID tag anti-collision," IEEE Transactions on Parallel and Distributed Systems, vol. 24, no. 1, pp. 19-31, 2013. 
[10] X. Yang, H. Wu, Y. Zeng, and F. Gao, "Capture-aware estimation for the number of RFID tags with lower complexity," IEEE Communications Letters, vol. 17, no. 10, pp. 1873-1876, 2013.

[11] $\mathrm{H}$. Wu and Y. Zeng, "Bayesian tag estimate and optimal frame length for anti-collision aloha RFID system," IEEE Transactions on Automation Science and Engineering, vol. 7, no. 4, pp. 963-969, 2010.

[12] Y. Li, H. Wu, and Y. Zeng, "Signal coding in physical layer separation for RFID tag collision," in Proceedings of the 2019 International Conference on Wireless Communication, Network and Multimedia Engineering (WCNME 2019), Guilin, China, 2019.

[13] Y. G. Kim and H. Vinck, "Anticollision algorithms for FM0 code and miller subcarrier sequence in RFID applications," IEEE Transactions on Vehicular Technology, vol. 67, no. 6, pp. 5168-5173, 2018.

[14] X. Lu and J. Li, "New Miller codes for run-length control in visible light communications," IEEE Transactions on Wireless Communications, vol. 17, no. 3, pp. 1798-1810, 2018.

[15] Y. C. Hung, M. M. Kuo, and C. K. Tung, "High-speed CMOS chip design for Manchester and Miller encoder," in 2009 Fifth International Conference on Intelligent Information Hiding and Multimedia Signal Processing, pp. 538-541, Kyoto, Japan, 2009.

[16] C. Angerer, R. Langwieser, and M. Rupp, "RFID reader receivers for physical layer collision recovery," IEEE Transactions on Communications, vol. 58, no. 12, pp. 3526-3537, 2010.

[17] L. Zhang, W. Xiang, and X. Tang, "An adaptive anti-collision protocol for large-scale RFID tag identification," IEEE Wireless Communications Letters, vol. 3, no. 6, pp. 601-604, 2014.

[18] S. K. Wijayasekara, S. Nakpeerayuth, R. Annur et al., "A collision resolution algorithm for RFID using modified dynamic tree with Bayesian tag estimation," IEEE Communications Letters, vol. 22, no. 11, pp. 2238-2241, 2018.

[19] X. Tan, W. He, F. Lingzhi, J. Wang, H. Min, and D. W. Engels, "Collision detection and signal recovery for UHF RFID systems," IEEE Transactions on Automation Science and Engineering, vol. 15, no. 1, pp. 239-250, 2018.

[20] C. Bowick, RF Circuit Design, Elsevier science \& technology, Oxford, UK, 2nd edition, 2007.

[21] L. Kong, L. He, Y. Gu, M.-Y. Wu, and T. He, "A parallel identification protocol for RFID systems," in IEEE INFOCOM 2014 - IEEE Conference on Computer Communications, pp. 154162, Toronto, ON, Canada, 2014.

[22] O. Jiajue, L. Mo, and Y. Zheng, "Come and be served: parallel decoding for COTS RFID tags," in Proceedings of the 21st Annual International Conference on Mobile Computing and Networking, pp. 500-511, Paris, France, 2015.

[23] X. Huang, G. Dolmans, H. de Groot, and J. R. Long, "Noise and sensitivity in RF envelope detection receivers," IEEE Transactions on Circuits and Systems II: Express Briefs, vol. 60, no. 10, pp. 637-641, 2013.

[24] F. Changxin, Principles of Communications, House of Electronics Industry, Beijing, China, 2nd edition, 2012.

[25] R. Lyons, Understanding Digital Signal Processing, Prentice Hall, New Jersey, United States, 3rd edition, 2010.

[26] Y. T. Toh, A. Niknejad, and B. Nikolic, "BPSK demodulation for RF applications," Technical Report No. UCB/EECS-201791, Electrical Engineering and Computer Sciences University of California at Berkeley, 2017, http://www2.eecs.berkeley.edu/Pubs/TechRpts/2017/EECS-2017-91.html. 